\title{
Conoscere il passato per capire il presente e orientare il futuro
}

\author{
di Elio Borgonovi
}

In una prospettiva storica, la pandemia Covid-19 è considerata da molti come un drammatico incidente di percorso, lungo una traiettoria di evoluzione del progresso scientifico, tecnologico, economico e sociale. A oggi però non è così sicuro che si tratti solo di un incidente di percorso in quanto potrebbe trattarsi di una rottura rispetto alle traiettorie precedenti. Spetterà alla storia dire se sarà vera rottura e se potrà essere considerata positiva o negativa, evolutiva o involutiva, rivoluzionaria o riformista. Giudizi che peraltro dipenderanno dai valori e dai criteri con cui gli storici futuri analizzeranno questo periodo.

Chi vive tuttora questa fase può fare analisi di breve periodo richiamandosi a quando già affermava Tucidide (filosofo, storico, condottiere greco del IV secolo a.C.), per il quale bisogna "conoscere il passato per capire il presente e orientare il futuro", senza dimenticare il detto popolare secondo cui "del senno del poi son piene le fosse".

Tralasciando le polemiche sulla più o meno tempestiva e trasparente comunicazione da parte della Cina, dove tutto è partito, è certo che in Italia, ma anche in altri Paesi europei, già a fine dicembre 2019 e sicuramente a gennaio 2020, si erano manifestate in vari ospedali "polmoniti anomale" che non rispondevano alle terapie consolidate; tra l'altro, in numero elevato rispetto alla media di una stagione invernale non particolarmente fredda. Di fronte a queste situazioni non era scattato il classico campanello d'allarme, tipico di chi ha un approccio scientifico, secondo cui date certe evidenze occorre ricercare spiegazioni. Le evidenze erano polmoniti anomale che non rispondevano alle terapie consolidate, ma pochi hanno avuto dubbi o sono andati alla ricerca delle cause.

Nemmeno dopo il lockdown di Wuhan e la dichiarazione dell'OMS di allarme pandemia a fine febbraio 2020, nei Paesi europei erano stati fatti approfondimenti di queste situazioni sanitarie, né erano state adottate particolari misure precauzionali. A tal proposito si vuol mettere in evidenza non i comportamenti di singoli medici, ma il fatto più generale che nei moderni sistemi di salute, basati sulle conoscenze scientifiche e tecnologiche molto sofisticate, si era atrofizzata la funzione fondamentale d'individuare segnali premonitori di qualcosa di strano che stava avanzando sul fronte della salute pubblica.

Anche gli scienziati e gli organismi, che si occupano di malattie trasmissibili, epidemie e pandemie, non sono stati concordi nella fase che può essere definita di "incubazione" non solo del virus, ma anche delle informazioni e delle comunicazioni. Pochi avevano percepito la pericolosità globale di questo virus, senza parlare di quanto è accaduto a Wuhan, dove sono stati tacitati in vario modo medici, che avevano compreso la gravità della pandemia e avevano lanciato i primi allarmi.

Inoltre gli esperti a livello internazionale e nei vari Paesi hanno avuto diverse posizioni: difronte a un virus nuovo non era facile prevedere i meccanismi di trasmissione. Tuttavia quella parte di "società della conoscenza", come da alcuni anni è stata definita, che si occupa di malattie trasmissibili, di fronte a ciò che avveniva a Wuhan, avrebbe potuto/ dovuto pensare e fare qualcosa di più rispetto alla dichiarazione di semplice allarme di rischio pandemia. Poiché le epidemie, e ancor più le pandemie, si trasmettono tra persone (con contatto fisico, con liquidi biologici, con il respiro) si sarebbe potuto ben immaginare che i passeggeri dei voli diretti o con scali intermedi da Wuhan o dalla provincia di mezzo effettuata e la sua messa a disposizione di terzi, sia in forma gratuita sia a pagamento. 
Hubei, potevano essere un potente veicolo di trasmissione globale in breve tempo. Invece, per lo meno fino al 21 febbraio 2020, in Italia la comunicazione si è concentrata solo sui due cittadini cinesi ricoverati all'ospedale Spallanzani di Roma o sui concittadini italiani da far rientrare da Wuhan. Inoltre, tra i virologi o esperti di malattie infettive vi era chi parlava ancora di epidemia solo un poco più grave di quelle conosciute. Anche coloro che già avevano lanciato allarmi su una maggiore gravità, salvo rari casi, non avevano suggerito misure particolarmente restrittive riguardo ai comportamenti, ai contatti sociali e all'utilizzo di mascherine, tema sul quale si sono sprecate non poche polemiche. Non è possibile sapere quanti e quali esponenti del mondo scientifico abbiano dato, in questa prima fase, indicazioni ai decisori politici, ma è indubbio che è parsa chiara la differenza tra sistemi democratici e autoritari. Nei primi era oggettivamente difficile prendere drastiche misure di prevenzione, come quello che poi è diventato il distanziamento sociale (sarebbe stato meglio distanziamento fisico per non accentuare le conseguenze delle disuguaglianze), prima che la diffusione del virus diffondesse la paura da un lato e il senso di responsabilità dall'altro.

Tutti ricordano i contrasti sul rischio di blocco di attività economiche e sulla alternativa tra tutela della salute e depressione economica fino al 7 marzo 2020, per non parlare dei dibattiti su eventi sportivi, con o senza la presenza dei tifosi, dalla seconda metà di febbraio fino alla prima settimana di marzo 2020. Il clima di incertezza, confusione, mancanza di chiari indirizzi da parte degli esperti, e non solo dei politici, aveva portato prima a posporre manifestazioni per poi cancellarle definitivamente. Tra i tanti sport si citano, per esempio: il rinvio delle partite di calcio in alcune città del Nord Italia e quelle invece giocate in città del Sud nel weekend 29 febbraio/ $1^{\circ}$ marzo, quelle giocate senza pubblico a porte chiuse nel weekend 7-8 marzo e le partite di Coppe Europee giocate con il pubblico normalmente sugli spalti o accalcati in migliaia fuori dagli stadi tra fine febbraio e i primi di marzo (Liverpool/Atletico Madrid, Paris Saint-Germain) Borussia Dortmund). Poi ancora, i rinvii delle ultime gare della Coppa del mondo di sci alpino, la sospensione di tutti gli eventi ciclistici (compresi i grandi giri di Italia, tour de France), dei Campionati di motociclismo e Formula1 quando si era praticamente ai nastri di partenza, addirittura il giorno prima delle prove ufficiali.

Nonostante gli studi e le ricerche sugli "eventi sentinella" per prevenire e ridurre errori e rischi clinici, gli studi e le ricerche sui segnali deboli nel campo della competitività e delle strategie aziendali, i sistemi di salute occidentali si sono fatti trovare impreparati. Oggi sono chiare le conseguenze di tale situazione, quindi per il futuro occorreranno politiche e comportamenti idonei a cogliere $i$ segnali deboli e gli eventi sentinella/premonitori, non tanto e non solo di future pandemie, ma anche di altre possibili emergenze globali. Passando al piano dell'assistenza, quando l'epidemia si è manifestata con la massima virulenza, va ricordato un punto di debolezza nel quale si è trovato il SSN. Infatti da almeno 20 anni anche nel nostro Paese sono state sviluppate analisi e proposte che avevano come fondamento concetti quali integrazione ospedale/territorio, continuità assistenziale, rafforzamento delle cure primarie e intermedie, percorsi diagnostico-terapeutico-assistenziali, presa in carico di pazienti con cronicità, disabilità o fragilità, centralità del paziente.

Mentre le teorie e le ricerche si sono sviluppate nella direzione della complessità, le politiche sono andate in senso contrario, ossia hanno assunto una prospettiva riduzionistica e della frammentazione. Vanno considerate tali le politiche di contenimento della spesa, di riduzione dei posti letto, di aggregazione di presidi ospedalieri e di blocco del turnover del personale, non solo come condizioni per il recupero di efficienza, ma in molti casi anche come condizioni per stimolare il miglioramento dell'appropriatezza e dell'efficacia.

Esistono scritti, prese di posizioni, provvedimenti e documentazioni che possono chiarire le diverse posizioni di studiosi, decisori politici e vertici di organismi tecnici a livello internazionale, nazionale e regionali, manager di aziende sanitarie, opinionisti, tribunali del malato e associazioni pazienti. Serve a poco dire oggi chi aveva ragione e chi aveva torto, serve invece capire che, nella fase critica del contagio, le diverse conseguenze, in termini di contagiati, ricoverati in terapia intensiva e morti, sono state influenzate anche dal grado di coordinamento tra diverse componenti del sistema. La risposta è stata migliore dove il coordinamento è stato più rapido e più forte, ha lasciato a desiderare dove il coordinamento è, invece, mancato o è stato debole.

La velocità e l'efficacia nell'affrontare quello che tutti hanno definito come tsunami sono state influenzate dalla capacità di affrontare, in modo più o meno unitario e organico, i seguenti aspetti: 
- comprensione della gravità del fenomeno e delle modalità di diffusione del contagio;

- individuazione dei pazienti sintomatici e asintomatici tramite tamponi (testing);

- modalità per ricostruire e tracciare i contatti (tracing);

- approvvigionamento dei dispositivi di protezione individuale e di attrezzature per processare i tamponi;

- aumento dei posti letto per terapie intensive, riconversione di reparti o interi ospedali per pazienti Covid-19 (treatment);

- separazione dei percorsi di accesso ai pronto soccorso, alle strutture sanitarie e alle aree dedicate a pazienti Covid-19;

- informazione e indirizzi di comportamento per medici, infermieri e vari operatori che hanno agito in diversi contesti;

- informazione ai cittadini su isolamento volontario, e in seguito obbligatorio, cure a domicilio in presenza di sintomi Covid-19 compatibili, attivazione "fase 2" ecc.

Una cosa oggi è certa. Tutti si sono accorti che la salute, come la libertà, è un bene diverso da tutti gli altri, in quanto si apprezza quando si perde. In passato, questa percezione toccava solo una parte della popolazione, quella che si ammalava, a volte per periodi brevi, per esempio in caso di incidenti o di patologie acute, passati i quali, non di rado, le persone dimenticavano questa verità. Altre volte in modo continuativo per chi è colpito da patologie croniche e degenerative. Oggi tutti si sono accorti di questa verità in quanto i comportamenti per tutelare la salute hanno condizionato pesantemente:

- i rapporti sociali (lockdown e distanziamento);

- i processi educativi dell'istruzione e della formazione (scuole e università), le attività economiche, l'organizzazione del lavoro e la distribuzione della ricchezza (con l'emergere di nuove disuguaglianze e povertà e con l'aggravamento di quelle già esistenti);

- il commercio internazionale, con la necessità di ripensare al modello di globalizzazione pre-Covid-19.

Per il futuro ne deriva un orientamento: la tutela della salute dovrà diventare ancor più un asse portante e una filiera fondamentale per una nuova economia. Alcune produzioni di beni collegati a questa filiera dovranno essere considerate strategiche per evitare di trovarsi nella situazione di scarsità o divieti alle esportazioni, nelle quali si sono trovati nei mesi scorsi diversi Paesi, tra cui il nostro. Pertanto la specializzazione internazionale delle produzioni non potrà essere governata solo da valutazioni di convenienza economica e dalla logistica distributiva, ma anche da criteri di autonomia e indipendenza. Per esempio, le gare pubbliche per la fornitura di mascherine per uso chirurgico, prima di Covid-19, erano state assegnate al prezzo di 8-10 centesimo di euro, che aveva messo fuori mercato non solo i produttori italiani ma tutti quelli del mondo occidentale. Nel periodo di blocco delle forniture, lo Stato e le Regioni hanno acquistato a prezzi di vari euro, quando addirittura non sono incappati in varie truffe, con pagamenti anticipati per forniture mai ricevute, e i cittadini, in molti casi, hanno acquistato le mascherine a prezzi di decine di euro. Passata la fase critica il Commissario straordinario ha imposto un prezzo controllato di 50 centesimi in farmacia e in altri punti vendita autorizzati. Anche in questo caso approcci riduzionistici, che consideravano unicamente l'economia di scala, l'efficienza, la riduzione dei costi o l'aumento dei profitti, che si sono rivelati vincenti per 20-30 anni, hanno creato le condizioni per una frantumazione dei sistemi economici che sarà difficile ricomporre.

Oggi si comprendono, anzi si vivono, le conseguenze negative di approcci riduzionistici del passato fondati su logiche dei due tempi, del prima e del dopo. Nel caso del SSN, prima, il recupero dell'equilibrio finanziario tramite il contenimento della spesa e, dopo, la preoccupazione per i potenziali effetti negativi sul piano della salute; prima, il rafforzamento dell'ospedale con tecnologie avanzate e, dopo, il potenziamento dell'assistenza territoriale; prima, la priorità alle patologie acute gravi e, dopo, l'assistenza alle malattie rare e l'assistenza alle presone con malattie croniche o degenerative; prima, l'assistenza sanitaria e, dopo, l'assistenza socio-sanitaria nelle RSA o socio-assistenziale con interventi dei Comuni separati da quelli sulla salute. Covid-19 ha dimostrato che la logica dei due tempi distinti ha quasi sicuramente causato una più elevata mortalità. Abbiamo anche imparato che, invece di rincorrere il virus, biso- 
gna attuare strategie di sbarramento della sua corsa. All'inizio della pandemia, l'Italia aveva un numero limitato di posti letto per terapia intensiva, poco più di 5.300. La rincorsa ha consentito in tempi brevi di aumentare il numero di posti letto di terapia intensiva, soprattutto nelle regioni che hanno avuto una maggiore pressione di casi gravi. Tutti ricordano i giorni drammatici di marzo e inizio aprile quando veniva comunicato che in alcune regioni e province si era vicini alla saturazione dei posti letto per terapie intensive e veniva consigliato ai medici di medicina generale di curare i pazienti a domicilio. In seguito il sollievo percepito quando giornalmente veniva annunciata la diminuzione dei pazienti ricoverati in terapia intensiva o comunque ricoverati in ospedale. È riuscita la manovra di alzare le barriere di fronte all'onda crescente, ma è stato pagato un prezzo elevato in termini di morti.

Queste drammatiche situazioni sono state vissute anche nei Paesi dove Covid-19 ha colpito più duramente, come Spagna, UK, USA e da metà maggio anche in America Latina. In futuro occorrerà una strategia diversa o almeno più bilanciata. Adeguare il numero di posti letto di terapia intensiva per avere un maggiore margine di accoglienza di pazienti gravi in caso di necessità e, contemporaneamente, predisporre linee guida e interventi con l'obiettivo di "abbassare l'onda". Tipicamente interventi di una più efficace assistenza a domicilio o comunque con modalità che prevengano l'aggravamento. Questo insegnamento sarà utile per affrontare le previste e probabili onde del virus, ma dovrà essere tenuto presente anche per affrontare le conseguenze economiche di Covid-19. Il controllo della diffusione del virus, dopo l'uscita dal lockdown, è stato condizionato dal comportamento più o meno responsabile delle persone con riguardo alle misure preventive e da quello delle aziende con riguardo all'adozione di sistemi di tutela dei lavoratori.

Sul piano economico bisogna evitare di ripetere l'errore di rincorrere i disastri causati da Covid-19 con la logica dei due tempi, prima pensare ai sussidi e poi alla ripartenza. Ovviamente, i sussidi sono stati e saranno necessari per alcuni mesi, a condizione che i soldi promessi arrivino velocemente alle famiglie e nelle casse delle imprese. Tuttavia usciranno meglio dalla crisi e con una ripresa più rapida e sostenibile, le regioni e i Paesi che, invece di rincorrere la disoccupazione e le nuove povertà, saranno riusciti a reinventare nuove attività per creare occupazione, in condizioni tutelate rispetto al permanere del virus, in attesa del vaccino. Occorrerà puntare tempestivamente sull'innovazione scientifica e tecnologica, sui sistemi educativi e di formazione orientati a nuovi profili professionali per accelerare l'evoluzione verso l'economia della conoscenza e l'accumulo di capitale umano. Occorrerà pensare a nuovi modelli di business in grado di valorizzare lo smart working e orari di lavoro flessibili per ridurre l'affollamento dei mezzi pubblici, senza trascurare interventi finalizzati a ridurre l'onda della povertà e dell'assistenzialismo.

Il nostro Paese, più di altri, ha bisogno di evitare un nuovo circolo vizioso secondo cui:

eccesso di cautela e misure rigide per tutelare la salute $\rightarrow$ blocco o difficoltà per le attività economiche $\rightarrow$ fallimenti di imprese $\rightarrow$ aumento disoccupazione e povertà $\rightarrow$ sussidi pubblici alle imprese e alle famiglie $\rightarrow$ aumento del debito pubblico $\rightarrow$ basso tasso di crescita $\rightarrow$ permanere di elevata disoccupazione e povertà.

Mentre nel periodo di massima intensità della pandemia tutti, compresi i negazionisti della prima ora, hanno accettato di considerare la salute prioritaria rispetto all'economia, per il futuro occorrerà evitare approcci altrettanto riduzionistici che rallentano la ripresa. Nella fase di massima emergenza è stata giusta la paura, ora, e nel periodo di convivenza con Covid-19, occorrerà mantenere alcuni comportamenti di attenzione, ma come ha affermato il presidente Franklin Delano Roosevelt, a seguito della crisi del 1929, l'unica cosa di cui dobbiamo avere paura è la paura stessa. In particolare occorrerà evitare la logica del prima e del dopo con riguardo alle seguenti problematiche:

- puntare tutto, o molto, in termini di finanziamenti di sostegno alla ricerca di terapie efficaci e del vaccino per Covid-19, prima, riducendo però significativamente i finanziamenti e il sostegno alla ricerca in altri ambiti di salute che sono causa di patologie gravi e di morti in numero altrettanto rilevante, dopo;

- preoccuparsi della pandemia di Covid-19 che ha colpito il cosiddetto primo mondo, prima, dimenticando altre pandemie più o meno locali che non toccano direttamente il mondo cosiddetto progredito, dopo; 
- mantenere o ripristinare in caso di successive ondate di infezioni misure rigide di distanziamento sociale, prima, trascurando gli effetti che esse possono avere su altri condizioni psico-fisiche di salute, dopo;

- preoccuparsi di contenere la diffusione delle infezioni da Covid-19 tra la popolazione generale, prima, senza predisporre soluzioni a favore di persone con disabilità o in condizioni di fragilità, dopo;

- aumentare il numero di posti letto per terapia intensiva, oppure rafforzare i presidi territoriali, prima, dimenticando che la soluzione corretta consiste nell'integrazione e nel coordinamento dei diversi livelli di intervento, dopo;

- aumentare il numero di personale sanitario, prima, dimenticando che è la formazione professionale e umana che consentirà una più elevata qualità dell'assistenza, dopo;

- riproporre eventualmente sussidi e sostegni alle famiglie e alle imprese, prima, invece che cogliere l'occasione per ripensare in modo organico a misure di sostegno dell'occupazione, e più in generale del sistema di welfare, e a una strategia del Paese di sostegno a filiere economiche innovative e competitive, dopo;

- considerare un maggiore intervento pubblico nelle fasi di emergenza come condizione necessaria e sufficiente per uscire dalla crisi, prima, dimenticando che in futuro si potrà uscire da crisi drammatiche e strutturali solo tramite una stretta e convinta collaborazione/partnership pubblico-privato, dopo.

La logica di conoscere il passato per capire il presente e orientare il futuro deve applicarsi anche alla sfera dei sentimenti, sistemi politici, sistemi istituzionali, relazioni internazionali.

Sul piano dei sentimenti va ricordato che all'iniziale sottovalutazione sono poi subentrati la grande paura, i grandi dolori e i sentimenti di riconoscenza verso gli eroi che sono stati in prima linea. Occorrerà aiutare sul piano psicologico coloro che hanno vissuto l'enorme fatica e lo stress della battaglia in prima linea, faccia a faccia con il dolore, la morte. Nei prossimi mesi sarà necessario anche aiutare coloro che hanno sofferto maggiormente sul piano psicologico sia per il lockdown sia per disoccupazione e povertà. Occorreranno interventi che aiutino molte persone e intere comunità a elaborare il lutto. Occorrerà aiutare soprattutto i giovani, ma non solo, a capire che non si può ritornare alla vita precedente e alla sottovalutazione di un nemico invisibile, ma che resterà ancora pericoloso. Occorrerà diffondere informazioni scientifiche, meno contraddittorie e più misurate, per mettere in guardia, senza eccessivi allarmismi ma nemmeno banalizzazioni, contro altri nemici della salute che potranno presentarsi in futuro. Non bisognerà sottovalutare i rischi quando questi nemici si presenteranno camuffandosi da nemici poco potenti. Occorrerà evitare che gli eroi di oggi diventino gli accusati di domani. Già sono state avviate cause e inchieste nei confronti di medici e responsabili di strutture che dovranno avere il giusto corso seguendo i crismi della giustizia, ma che non dovranno trasformarsi in una nuova ondata di giustizialismo. Occorrerà valutare e giudicare i comportamenti non con "il senno del poi", ossia passata la bufera, ma considerando la bufera nella quale molti hanno dovuto decidere e agire. La giustizia è giusta quando è equa ed evita gli estremi: del giustizialismo, ossia della ricerca del colpevole a ogni costo, e del giustificazionismo, ossia della depenalizzazione o "scudo per causa di emergenza" che spesso serve a difendere più $i$ vertici, politici o tecnici che siano, che non coloro che sono stati in trincea.

Sul piano politico, dopo aver assistito a forme di presenzialismo e di protagonismo, di cui nessuno sentiva il bisogno, sarebbe auspicabile o desiderabile evitare la lotta di "tutti contro tutti", che purtroppo però è già partita nel nostro Paese, come in alcuni altri. L'unico effetto sarebbe quello di delegittimare ulteriormente una funzione di cui invece la società ha molto bisogno, purché sia esercitata con il ritorno alla cultura di chi si preoccupa della res publica. Una funzione politica "alta e responsabile" sarà chiamata ad affrontare nuovi rapporti tra salute/economia/ambiente/ sostenibilità/democrazia. L'orientamento per il futuro riguarda anche, se non soprattutto, i cittadini. Se è vero che ogni Paese ha la classe politica che si merita, i cittadini si troveranno di fronte a un'alternativa. Dare il loro consenso a persone con preparazioni ed esperienze solide, molto collegate ai problemi reali, che nel gergo popolare significa badare alla sostanza e non alle apparenze, coerenti nelle diverse fasi e ruoli della loro vita e nelle prese di posizione e capaci di decidere tramite l'attivazione di processi partecipativi a vari livelli. Oppure, seguendo la cultura "antisiste- 
ma", dare il proprio consenso ad affabulatori, a chi fa facili promesse, a chi cavalca le emozioni sociali, a chi costruisce la propria immagine creando nemici più o meno immaginari, pericolosi e potenti. Si sente parlare della necessità di "rifondare la politica" o di sostituire la classe politica, ma il vero problema è quello di diffondere nella popolazione $i$ semi di una cultura che consenta di far crescere la "buona politica".

Sul piano istituzionale, occorrerà rivitalizzare il principio dell'equilibrio, o meglio del bilanciamento tra $i$ diversi poteri dello Stato. Ci sono segnali, a livello mondiale, di uso strumentale della situazione di emergenza e di crisi per orientare i sistemi istituzionali verso poteri forti, se non addirittura autoritari. Sarebbe una soluzione "riduzionistica" perché non in grado di affrontare, in modo sostenibile nel lungo periodo, le relazioni tra potere-libertà, obblighi-consenso, efficienza economica-sostenibilità sociale. Soluzioni autoritarie, dell'uomo forte, potrebbero avere successo nel breve periodo (alcuni decenni sono un breve periodo rispetto alla storia), ma sicuramente non sarebbero sostenibili nel medio-lungo periodo. Restando all'interno del nostro Paese occorrerà rifuggire dalla tentazione della centralizzazione intesa in senso generico. Certamente Covid-19 ha riproposto in termini drammatici il tema del rapporto tra unità del SSN e autonomie regionali. Un rapporto che dovrà essere ridefinito non sulla base di "modelli astratti" utili e necessari per situazioni di emergenze nazionali e globali, ma sulla base di analisi e valutazioni della "concreta realtà" ed "esperienze", positive e negative, maturate prima e durante Covid-19. Non va dimenticato che un potere centrale forte può essere positivo se ben esercitato in modo corretto e da persone con elevate conoscenze e competenze professionali, manageriali e di policy-making, ma diventa dirompente in senso negativo quando, esercitato senza queste condizioni, ostacola le buone pratiche che pure sono state presenti, in varie regioni. Per cercare soluzioni migliorative bisogna confrontarsi con questo dilemma: che cosa sarebbe successo in tutto il Paese se un sistema centralizzato si fosse comportato come le regioni considerate virtuose e invece che cosa sarebbe successo se questo potere centrale avesse scelto di comportarsi come le regioni considerate "meno virtuose"? Ad analisi in tempi più sereni è demandato il compito di definire virtuoso e meno virtuoso. Però si ricorda quanto è successo in UK, Svezia, USA e Brasile, dove vi sono stati un potere o comunque indirizzi centrali uniformi e forti. È auspicabile un rafforzamento del potere centrale in grado di sostenere organici programmi di ricerca sui temi della salute, di dare chiari indirizzi compatibili con la situazione del Paese, attivare processi di stimolo dell'innovazione dal basso e di raccolta e diffusione delle buone pratiche di innovazione sul piano assistenziale, organizzativo, manageriale, intervenire con un'azione di supporto e non con vincoli nelle realtà con più bassi livelli assistenziali e alti livelli di efficienza, attivare rapidamente il coordinamento in caso di emergenze nazionali e internazionali. Sarebbe dannosa una centralizzazione che volesse imporre modelli assistenziali e organizzativi rigidi, presentati come "ottimali" sul piano astratto, imporre soluzioni uniformi negando le differenze che caratterizzano i problemi di salute, standardizzare ciò che non è standardizzabile, imporre controlli burocratici e contabili che appesantiscono la gestione delle ASL, privilegiare le responsabilità formali derivanti dalla sfiducia nei confronti di Regioni e di ASL rispetto a sistemi basati sul principio della fiducia che consiste nel riconoscere autonomia e responsabilizzare su risultati di salute e sull'equilibrio complessivo e non di singole voci di spesa. Un tema da approfondire da parte di chi si appresta a rivedere l'intero impianto del SSN che dovrà considerare i principi di universalità, solidarietà, equità tenendo conto non della realtà di oggi ma proiettandoli nei prossimi 10-20 anni.

Sul piano delle relazioni internazionali, Covid-19 ha generato scombussolamenti non marginali. La Cina, che è stata l'origine di tutto, nella fase post-pandemia ha intensificato politiche di assistenza, invio di materiale e di supporto a Paesi africani e, più velatamente ma non meno decisamente, a Paesi europei. Una politica che implica una modifica della strategia commerciale ma anche del ruolo di influenza, il cosiddetto soft power. Negli Stati Uniti il presidente Trump ha accentuato la politica di America first basata sulla creazione di nemici esterni e interni (Cina, OMS, dimostrazioni antirazziali) per cercare di compattare il proprio elettorato, in vista delle elezioni di novembre 2020. Il crollo del prezzo del petrolio ha e avrà impatto pesante sui Paesi produttori, ma anche sulle politiche riguardanti le energie rinnovabili. L'Europa si è mossa in ordine sparso con Paesi che hanno chiuso le frontiere a persone e beni considerati strategici, nonostante gli appelli all'azione unitaria e coordinata da parte della Commissione e della BCE per 
le rispettive competenze. Alcuni segnali positivi, a maggio, sono venuti dall'approvazione di politiche di utilizzo del MES - Meccanismo Europeo di Stabilità alla sola condizione di utilizzo per interventi, diretti e indiretti, nel settore della tutela della salute, dal programma SURE - Support to mitigate Unemployment Risks in an Emergency contro la disoccupazione e dal Recovery Fund (Next Generation Fund), il Fondo per la ripartenza proposto dalla Commissione europea, che dovrà essere approvato nei prossimi mesi. Si vedrà se i contrasti tra Paesi che si autodefiniscono frugali e Paesi più colpiti dalla pandemia potranno essere superati. In caso affermativo la costruzione europea sarà rafforzata, in caso negativo si rischia una involuzione storica con conseguenze imprevedibili. I Paesi poveri, abbandonati a se stessi, stanno diventando sempre più poveri e quindi terreno fertile per nuove forme di colonizzazione. Gli organismi internazionali hanno lanciato allarmi su carestie provocate dall' invasione di cavallette, siccità e altre cause che rischiano di raddoppiare il numero di persone sotto la soglia della povertà assoluta. Tutti appelli rimasti, però, in gran parte inascoltati da Paesi, cosiddetti progrediti, impegnati, prevalentemente, a sviluppare terapie e vaccini antiCovid-19 e a rimettere in sesto le proprie economie.

Facendo tesoro della storia si può dire che da crisi profonde, quale è stata indubbiamente quella causata da Covid-19, persone, istituzioni pubbliche e private, Paesi e ordine globale non escono mai uguali, ma migliori o peggiori. Poiché nessuno ha il potere di cambiare, da solo, il mondo, ma tutti abbiamo il potere/dovere di cambiare quella piccola, piccolissima o infinitesimale parte del mondo su cui abbiamo influenza, ognuno di noi ha la responsabilità di operare affinché prevalga la prima alternativa. 